\title{
Empirische Fundierung von Zentrale-Orte-Konzepten - Vorschlag für ein idealtypisches Vorgehen
}

\author{
Thomas Terfrüchte ${ }^{1}(\mathbb{D}) \cdot$ Stefan Greiving $^{2} \cdot$ Florian Flex $^{2}$
}

Eingegangen: 1. Dezember 2016 / Angenommen: 24. August 2017 / Online publiziert: 5. September 2017

(c) Springer-Verlag GmbH Deutschland 2017

Zusammenfassung Das Thema „Zentrale Orte“ erfreut sich sowohl in den Raumwissenschaften wie auch in der Praxis der Raumordnung (wieder) einer wachsenden Bedeutung. Zunehmend wird auf das Erfordernis einer empirischen Validierung von Zentrale-Orte-Konzepten abgestellt, damit die entsprechenden Festlegungen mit Zielqualität in Raumordnungsplänen nachvollziehbar begründet werden können. Es bleibt bisher aber offen, welche methodischen Anforderungen an diese Validierung geknüpft werden und wie eine Validierung im Einzelnen ablaufen sollte. Diese Fragen stehen im Kern dieses Beitrags, der vier mögliche Vorgehensweisen bei der empirischen Fundierung von Zentrale-Orte-Konzepten als unabdingbare Voraussetzung für Rechtssicherheit vorstellt und schließlich ein iteratives Vorgehen vorschlägt.

Schlüsselwörter Zentrale-Orte-Konzept .

Raumordnerisches Zielsystem - Raumordnerisches

Erfordernis $\cdot$ Operationalisierung $\cdot$ Validierung

Dr. Thomas Terfrüchte

thomas.terfruechte@tu-dortmund.de

Prof. Dr.-Ing. Stefan Greiving

stefan.greiving@tu-dortmund.de

Dr.-Ing. Florian Flex

florian.flex@tu-dortmund.de

1 Fachgebiet Raumordnung und Planungstheorie,

Technische Universität Dortmund,

August-Schmidt-Straße 6, 44227 Dortmund,

Deutschland

2 Institut für Raumplanung, Technische Universität Dortmund, August-Schmidt-Straße 10, 44227 Dortmund, Deutschland

\section{Empirical foundation of central-place-concepts - Recommendations for an ideal type approach}

\begin{abstract}
The topic of "central places" (again) steadily gains relevance in spatial science as well as in regional planning. Emphasising the requirement to verify central-place concepts empirically, corresponding aims of comprehensive regional planning can be justified in a reasonable way. It has so far remained open which methodological demands have to relate to this validation and how in particular these specifications should be fulfilled. These questions form the background for this article. It introduces four optional approaches towards the empirical foundation of central-place concepts as an indispensable prerequisite for legal certainty and proposes in the end an iterative approach as ideal type.
\end{abstract}

Keywords Central-place concept - Goals of spatial planning - Legal requirements in spatial planning . Operationalisation $\cdot$ Validation

\section{Einleitung}

In den Raumwissenschaften wie auch in der Praxis der Raumordnung erfährt das Thema „Zentrale Orte“ (wieder) wachsende Bedeutung. So stärkt die Ministerkonferenz für Raumordnung (MKRO) mit ihrer jüngsten Entschließung (MKRO 2016a) grundsätzlich die Steuerungswirkung von Zentrale-Orte-Konzepten und nimmt in den überarbeiteten Leitbildern der Raumentwicklung (MKRO 2016b) explizit 
Bezug darauf. ${ }^{1}$ Vor allem das Leitbild „Daseinsvorsorge sichern" im Kontext gleichwertiger Lebensverhältnisse ist hier zu nennen. Die Akademie für Raumforschung und Landesplanung (ARL) hat im Rahmen mehrerer Landesarbeitsgemeinschaften Arbeitsgruppen zur Fortentwicklung der Zentrale-Orte-Konzepte eingerichtet bzw. jüngst abgeschlossen. ${ }^{2}$ Das Bundesinstitut für Bau-, Stadt- und Raumforschung (BBSR) hat 2014 eine deutschlandweit vergleichende Studie zu den Zentrale-Orte-Konzepten der Länder veröffentlicht (Greiving/Winkel/Flex et al. 2014). Und schließlich nehmen zahlreiche obergerichtliche Urteile insbesondere im Zusammenhang mit der Steuerung des großflächigen Einzelhandels (Kongruenzgebot, Beeinträchtigungsverbot), der Siedlungsentwicklung oder dem kommunalen Finanzausgleich regelmäßig direkt Bezug auf die länderspezifischen Zentrale-Orte-Konzepte und fordern von den Landesraumordnungen entsprechende Reformen ein, die in einzelnen Bundesländern bereits durch Gutachten vorbereitet werden. Grundtenor des eingeforderten Reformbedarfs ist vor allem das Erfordernis der empirischen Fundierung raumordnerischer Festlegungen. ${ }^{3}$ Dem hat sich die MKRO (2016a) mit Punkt 8 ihrer Empfehlungen angeschlossen. Dies gilt insbesondere vor dem Hintergrund der mit der Festlegung Zentraler Orte verbundenen Einschränkung kommunaler Planungshoheit durch überörtliche Steuerungskonzepte - etwa in den Bereichen Siedlungsflächenentwicklung und großflächiger Einzelhandel ${ }^{4}$ - sowie die ohnehin bestehenden Anforderungen an Ziele

\footnotetext{
1 Die Ministerkonferenz für Raumordnung hat in ihrer Entschließung (MKRO 2016a) allerdings ein spezielles Begriffsverständnis - und zwar umgekehrt zur vorherrschenden Meinung in der Wissenschaft. Die Ministerkonferenz für Raumordnung definiert das Zentrale-Orte-System als normatives Ziel der Raumordnung und das Zentrale-Orte-Konzept als empirischen Analyserahmen. In der Wissenschaft hingegen ist vom deskriptiven Zentrale-Orte-System und normativen Zentrale-Orte-Konzept die Rede (vgl. etwa Blotevogel 2002a; Blotevogel 2005; Winkel/Greiving 2008; Flex 2015; Furkert 2015; Terfrüchte 2015; Greiving/Flex/Terfrüchte 2015). Dem Begriffsverständnis der Ministerkonferenz für Raumordnung schließen sich die Verfasser dieses Beitrags daher bewusst nicht an und sprechen von der Validierung von (normativen) ,Zentrale-Orte-Konzepten“.

2 Arbeitsgruppen der Landesarbeitsgemeinschaften Bayern (,Reform des Zentrale-Orte-Konzepts in Bayern"), Bremen/Hamburg/ Niedersachsen/Schleswig-Holstein (,Die Funktion der Ortskerne für die Stabilisierung Zentraler Orte"; vgl. LAG ARL 2014), Hessen/ Rheinland-Pfalz/Saarland (,Ausstattungsprofile Zentraler Orte unter veränderten Rahmenbedingungen - das Beispiel von Hessen, Rheinland-Pfalz, Saarland“; vgl. ARL 2013) und Nordrhein-Westfalen („Neuaufstellung des Zentrale-Orte-Konzepts in Nordrhein-Westfalen“; vgl. Greiving/Flex/Terfrüchte 2015 und Greiving/Flex 2016).

${ }^{3}$ Für Nordrhein-Westfalen finden sich solche Forderungen beispielsweise in ARL (2015: 3), Terfrüchte (2015: 246) oder Landtag Nordrhein-Westfalen (2015: $40 \mathrm{ff}$.).

${ }^{4}$ Die Steuerungswirkungen Zentraler-Orte-Konzepte unterscheiden sich erheblich zwischen den Bundesländern (vgl. ausführlich Greiving/Winkel/Flex et al. 2014).
}

der Raumordnung wie das Bestimmtheitsgebot oder das Willkürverbot.

Der vorliegende Beitrag stellt ein idealtypisches Vorgehen bei der empirischen Fundierung von Zentrale-Orte-Konzepten vor und ist wie folgt aufgebaut: Zunächst werden die angesprochenen Grundmerkmale Zentraler Orte definiert (Kapitel 2). Im Anschluss werden allgemeine methodische Überlegungen zur Validierung von Zentrale-OrteKonzepten angestellt, wobei insbesondere eine begriffliche und inhaltliche Trennung zwischen Monitoring, Evaluation, Controlling und Validierung erfolgt (Kapitel 3). Darauf basierend werden idealtypische Vorgehensweisen zur Validierung vorgeschlagen (Kapitel 4), bevor schließlich der Beitrag mit einem Fazit endet (Kapitel 5).

Die Abhandlung knüpft zudem unmittelbar an die 2015 in dieser Zeitschrift publizierte „Vergleichende Untersuchung der Zentrale-Orte-Konzepte in den Ländern und Empfehlungen zu ihrer Weiterentwicklung" von Greiving, Flex und Terfrüchte an (Greiving/Flex/Terfrüchte 2015). Die hier dargelegten Überlegungen zu einer empirischen Fundierung sind vor diesem Hintergrund auch als Beitrag zu verstehen, den dort angesprochenen ,planungspolitischen Kraftakt" (Greiving/Flex/Terfrüchte 2015: 296) auf einer substanziellen empirischen Basis aufsetzen zu können.

Zwei wesentliche Prämissen liegen dem Ziel des Artikels zugrunde: Erstens wird vorausgesetzt, dass eine größtmögliche Akzeptanz der Zentrale-Orte-Konzepte angestrebt wird. Dabei geht es weniger um die ohnehin erforderliche Beteiligung bei der Aufstellung von Raumordnungsplänen ( $\$ 10$ ROG), sondern um die Akzeptanz des Planungsprozesses, was vor allem bei überörtlichen Planungen mitunter besonders schwierig sein kann (vgl. Wiechmann/Terfrüchte 2013). Im Vordergrund steht daher ein transparenter Prozess, der, so Fürst (2010: 171), zugleich auch legitimierend und damit akzeptanzsteigernd wirke. Planung, so Fürst (2010: 171) weiter, wirke eben nicht nur durch die Ziele der Raumordnung, ,sondern auch durch den Prozess der Planerstellung“. Es reicht somit nicht aus, dass ein ZentraleOrte-Konzept (als Ziel der Raumordnung) überwiegend akzeptiert wird, sondern auch der Prozess der Erarbeitung im Sinne eines Verfahrenskonsenses.

Die zweite Prämisse betrifft das grundsätzliche raumordnerische Verständnis von Zentralen Orten. Denn es ist mitnichten der Fall, dass sämtliche Landesplanungsbehörden ein - zumindest in den Grundzügen - einheitliches Begriffsund Steuerungsverständnis haben. Schon die Frage, welche und wie viele Hierarchiestufen samt Zwischenstufen ein Zentrale-Orte-Konzept aufweist, wird höchst unterschiedlich beantwortet (Greiving/Flex/Terfrüchte 2015: 287). Vor diesem Hintergrund schlagen die Verfasser klar definierte Grundmerkmale für Zentrale-Orte-Konzepte bzw. Zentrale Orte vor, auf denen die empirische Fundierung fußt. 


\section{Merkmale und Funktionen Zentraler Orte}

Zentrale-Orte-Konzepte sind Bestandteil aller Pläne und Programme der Landes- und Regionalplanung der Flächenländer, was die Bedeutung für die deutsche Raumordnungspraxis unterstreicht. Zentrale-Orte-Konzepte sind insofern aber auch als Plural zu verstehen, da es das eine ZentraleOrte-Konzept nicht gibt bzw. aufgrund der Länderzuständigkeit de facto auch nicht geben kann. ${ }^{5}$ Vor diesem Hintergrund werden im Folgenden - aus Sicht der Verfasser - die wesentlichen Merkmale Zentraler Orte hergeleitet, die als ,Grundkonsens' eines übergeordneten Zentrale-Orte-Konzepts verstanden werden können. Somit wird auch dem Erfordernis der intersubjektiven Nachvollziehbarkeit innerhalb einer in sich konsistenten methodischen Vorgehensweise, die sich ein Plangeber zu eigen macht, genüge getan (Faßbender 2012: 87).

\subsection{Versorgungsfunktion und zentrale Einrichtungen}

Die Versorgungsfunktion Zentraler Orte wird durchgängig in allen Zentrale-Orte-Konzepten angesprochen. Sie ist die klassische Funktion Zentraler Orte im Sinne einer Versorgung der eigenen und der Umlandbevölkerung mit Gütern und Dienstleistungen (zentrale Versorgungseinrichtungen) (vgl. z. B. Christaller 1968: 22; Klöpper 1970: 3850; Blotevogel 2005: 1313). Es geht also vor allem um nicht ubiquitär verfügbare Einrichtungen hauptsächlich der Daseinsvorsorge. Insofern gehören weder technische Infrastrukturen, wie etwa der Ver- und Entsorgung, noch Dienstleistungen, die nicht auf den Endverbraucher ausgerichtet sind wie etwa Unternehmensberater, zu den zentralen Versorgungseinrichtungen. Die Versorgungsfunktion ist in der Praxis der Raumordnung und der Wissenschaft gleichermaßen gut erforscht bzw. diskutiert (vgl. Terfrüchte 2015: 98 ff.). Dies ist mitunter auch auf die drei wegweisenden Entschließungen der Ministerkonferenz für Raumordnung zu Zentralen Orten aus den Jahren 1968, 1972 und 1983 zurückzuführen, was sich vor allem darin zeigt, dass die in den Plänen und Programmen aufgeführten Versorgungseinrichtungen (in der Regel in Ausstattungskatalogen) sich überwiegend mit den von der Ministerkonferenz für Raumordnung vorgeschlagenen Einrichtungen decken (Terfrüchte 2015: 100). Während also die Versorgungsfunktion im Allgemeinen und auch die Einrichtungen (methodisch gesehen die manifesten Merkmale Zentraler Orte) im Speziellen als unstrittig gelten können, gibt es verschiedene methodische Ansätze, um von den Einrichtungen auf die Zentralität eines Ortes (methodisch gesehen das latente Merkmal Zentraler Orte)

\footnotetext{
${ }^{5} \mathrm{Zu}$ den Unterschieden in der Festlegungspraxis der Länder siehe insbesondere die Untersuchungen von Greiving, Winkel, Flex et al. (2014).
}

zu schließen (Niedzwetzki 1977: 81 ff.; Deiters 1978: 60 ff.; Flex 2015: 235 ff.; Terfrüchte 2015: 138 ff.).

\subsection{Entwicklungs-/Stabilisierungsfunktion}

Neben der Versorgungsfunktion ist die Entwicklungsfunktion ein zweites wesentliches Merkmal Zentraler Orte (vgl. z.B. Ganser 1977: 110 ff.). Sie seien, wie Blotevogel (2005: 1314) es ausdrückt, insbesondere für die oberen Hierarchiestufen ,stärker zu betonen“. Dies ist insofern folgerichtig, da Zentrale Orte in der Raumordnung auch als Entwicklungszentren für die mittel- und oberzentralen Versorgungsbereiche verstanden werden (Terfrüchte 2015: 91). Je mehr entwicklungsfördernde und je weniger entwicklungshemmende Merkmale ein Ort aufweist, desto eher ist er - so der dahinter liegende Gedankengang in der Lage, entsprechende Entwicklungs- oder Stabilisierungsimpulse für den jeweiligen Versorgungsbereich zu setzen. Welche Merkmale jedoch als förderlich und welche als hemmend für die (regionale) Entwicklung gelten können, ist wissenschaftlich - anders als für die Versorgungsfunktion - nur unzureichend erforscht. Während etwa demographische, ökonomische oder fiskalische Merkmale zwar unisono als relevant erachtet werden (vgl. z. B. Staack 1995: $121 \mathrm{ff}$; Henckel/Kolleck/Mittag et al. 2002: 93; Hesse 2010: 106f.; Junkernheinrich/Boettcher/Brand et al. 2011: 31; Flex 2015: 327 ff.; Terfrüchte 2015: 106; Wiechmann/Terfrüchte 2017: $21 \mathrm{ff} ., 72 \mathrm{ff}$.), besteht über die gewünschten Merkmalsausprägungen keine Einigkeit. Das Beispiel der kommunalen Schulden, die gemeinhin als entwicklungshemmend eingestuft werden, zeigt dies eindrücklich. So weisen Boettcher und Junkernheinrich (2010: 112) darauf hin, dass ,umfangreiche investive Schulden tendenziell eine hohe finanzielle Leistungsfähigkeit signalisieren“, weshalb primär Kassenkredite „das Kennzeichen einer besonders prekären Finanzsituation" (Boettcher/Junkernheinrich 2010: 19) seien. Es ist somit eine Differenzierung der Schuldenart erforderlich.

Gleiches gilt für die demographische Entwicklung: Terfrüchte (2015: 225) hat etwa für Nordrhein-Westfalen feststellen können, dass ein geringer Jugendquotient als hemmendes Merkmal gelten kann, im Umkehrschluss aber weder ein hoher Jugendquotient noch ein geringer Altenquotient als entwicklungsfördernd eingestuft werden können. Da bei der Entwicklungsfunktion somit - im Unterschied zur Versorgungsfunktion - insgesamt ein erhebliches Theoriedefizit attestiert werden kann (Terfrüchte 2015: $248 \mathrm{f}$.), verwundert es nicht, dass auch in diesem Bereich verschiedene methodische Ansätze existieren, um von den manifesten Merkmalen auf die latente Entwicklungsfähigkeit von Orten zu schließen. Vor allem aber wird die Entwicklungsfunktion in der Regel jenseits der ,klassischen ' Zentralitätsforschung erforscht (vgl. z.B. Staack 1995: 121 ff.; Henckel/ 
Kolleck/Mittag et al. 2002: 93; Hesse 2010: 106 f.; Junkernheinrich/Boettcher/Brand et al. 2011: 31; Wiechmann/ Terfrüchte 2017: 21 ff., $72 \mathrm{ff}$.). Die Verfasser dieses Beitrags plädieren aber eindeutig für eine Einbeziehung der Entwicklungsfunktion bei der empirischen Beschreibung und - noch wichtiger - bei der in die Zukunft gerichteten Festlegung Zentraler Orte. Wenn Orte als Zentrale Orte festgelegt werden, sollten solche, die es empirisch ermittelt gegenwärtig sind, dies auch künftig bleiben können, und solche, die es empirisch eigentlich noch nicht sind, dies im Planungszeitraum ,schaffen ' können. Aus diesem Grund erfolgt mitunter - wenn bislang auch eher zaghaft - in einigen Ländern eine Verknüpfung vom Zentrale-Orte-Konzept mit der regionalen Strukturpolitik und/oder dem kommunalen Finanzausgleich (Greiving/Winkel/Flex et al. 2014: 70 ff.).

\subsection{Bereichsbildungsfunktion}

Die Bereichsbildungsfunktion dient - gemäß der Zentrale-Orte-Theorie - der Mitversorgung von weiteren (Umland-)Gemeinden (Versorgungsbereich) durch die Zentralen Orte. In Verbindung mit der Entwicklungsfunktion kann der Versorgungsbereich zudem als der räumlich-funktionale Verflechtungsbereich gesehen werden, für den der Zentrale Ort Entwicklungs- bzw. Stabilisierungsimpulse setzen kann. Was somit theoretisch erforderlich ist, wird auch von der Rechtsprechung vor allem hinsichtlich des Kongruenzgebotes und des Beeinträchtigungsverbotes regelmäßig eingefordert: Zentrale Orte definieren sich über ihre überörtliche Bedeutung, die nur dann attestiert werden kann, wenn eindeutig benannt ist, wie und wo sich die Überörtlichkeit räumlich darstellt. Jeder Zentrale Ort braucht daher auch einen bestimmten - oder zumindest bestimmbaren - Versorgungsbereich. Andernfalls läuft die Steuerungswirkung der Zentrale-Orte-Konzepte ins Leere, denn Tragfähigkeits- oder Erreichbarkeitskriterien bleiben so lange funktionslos, wie keine ,räumliche Kulisse abgesteckt ist, innerhalb derer Erreichbarkeit und Tragfähigkeit gewährleistet werden soll“ (Terfrüchte 2015: 245). Die in vielen Ländern praktizierte Gleichsetzung Zentraler Orte mit politischen Gemeinden kann gleichwohl auch - aus theoretischer Sicht - sogenannte Selbstversorgerorte ermöglichen, sofern ein „Hauptort“ die übrigen Orte bzw. Stadt-/Ortsteile auf dem Gemeindeterritorium mitversorgt. Überörtlichkeit und Übergemeindlichkeit sind insofern auch sachinhaltlich voneinander $\mathrm{zu}$ trennen (vgl. auch Langguth 2011).

Ähnlich wie bei der Versorgungsfunktion besteht in der Literatur weitgehend Einigkeit darüber, was es bei der Abgrenzung von Versorgungsbereichen zu erforschen gilt: Die räumlich-funktionalen Verflechtungen zwischen Zentralen Orten und nicht zentralen Orten bzw. zwischen Zentralen Orten höherer Hierarchiestufen und Zentralen Orten nied- riger Hierarchiestufen (vgl. z. B. Klöpper 1970: 3854 ff.; Klöpper 1972: 261 f.; Körber 1972: 243 ff.; Heinritz 1977: $21 \mathrm{ff}$.). Methodisch sauber zählen sämtliche sich auf die Versorgungsfunktion der Zentralen Orte beziehenden Verflechtungen $\mathrm{zu}$ den zentralörtlich relevanten und insofern $\mathrm{zu}$ berücksichtigenden Verflechtungen. Aufgrund des erheblichen Erhebungsaufwandes wird jedoch üblicherweise mit Proxy-Indikatoren gearbeitet. So konnte etwa im Bereich der ambulanten medizinischen Versorgung festgestellt werden, dass Mitversorgungsbeziehungen signifikant mit Berufspendlerverflechtungen korrelieren (Czihal/von Stillfried/Schallock 2012: $9 \mathrm{ff}$.). Neben den Berufspendlerverflechtungen liegen in der kommunalen Statistik in der Regel auch Verflechtungen im Schülerverkehr vor, die erstens unmittelbar auf die Versorgungsfunktion (im Bildungsbereich) bezogen sind und zweitens auch als Proxy-Indikator für weitere Verflechtungsbeziehungen gelten können.

Im Gegensatz zum „Was?“ der Erforschung besteht über das „Wie?“ der Erforschung jedoch grundlegende Uneinigkeit. Dies rührt auch aus der - theoretisch mindestens fragwürdigen - Vorstellung, Zentrale Orte könnten a priori anhand ihrer Versorgungs- und gegebenenfalls Entwicklungsfunktion empirisch bestimmt werden, ohne die jeweilige ,Umlandbedeutung ' methodisch zu berücksichtigen. Bereichsbildung kann theorieimmanent und im Hinblick auf die Rechtsprechung nicht an - wie auch immer definierten - Schwellenwerten oder nach anderen Kriterien ermittelten Orten ansetzen. Vielmehr bedeutet Bereichsbildung die Fähigkeit, weitere Orte mitzuversorgen (zentralörtliche Gravitationswirkung) und tatsächlich auf sich ,zu vereinen'.

\subsection{Raumordnerisches Erfordernis}

Ganz abgesehen von der Auswahl der beobachtbaren Merkmale zur Versorgungs-, Entwicklungs-/Stabilisierungs- und Bereichsbildungsfunktion sowie einer geeigneten Methodik zur empirischen Beschreibung von Zentrale-Orte-Systemen handelt es sich beim Zentrale-Orte-Konzept um ein normatives Steuerungsinstrument der Raumordnung. Vorab sei dazu Folgendes in Erinnerung gerufen: Keine Gemeinde sollte alleine aus ihrer Ausstattung einen Anspruch auf eine zentralörtliche Funktionszuweisung ableiten können (Greiving/Glex/Terfrüchte 2015: 292). Dieser Forderung hat sich zuletzt auch die Ministerkonferenz für Raumordnung in der eingangs erwähnten neuen Entschließung zu Zentralen Orten angeschlossen: „Allein das Vorhandensein von zentralörtlichen Ausstattungsmerkmalen führt nicht $\mathrm{zu}$ einem Anspruch von Städten oder Gemeinden auf die Festlegung als Zentralem Ort einer bestimmten Stufe. Die Auswahl und Festlegung Zentraler Orte ist Ergebnis einer Abwägung auf Grundlage eines gesamträumlichen planerischen Konzepts“ (MKRO 2016a: 6). Eine substanzielle Versorgungsfunktion 
ist zwar eine in der Regel zu erfüllende, aber als solche nicht hinreichende Voraussetzung für eine entsprechende Funktionszuweisung. Die in den Plänen und Programmen der Länder oftmals bereits angesprochene Entwicklungsfunktion wiederum scheint eher eine die Versorgungsfunktion ergänzende oder erst ermöglichende Funktion zu sein, weshalb sie - für sich genommen - weder notwendig noch hinreichend ist. Die Bereichsbildungsfunktion wiederum ist für sich genommen - erforderlich, weist jedoch zugleich das größte normative Moment auf, da mit der Abgrenzung von Versorgungsbereichen oftmals räumlich-funktionale Verflechtungen erst politisch-planerisch motiviert forciert werden sollen. So ist es etwa dem Prinzip der Einräumigkeit der Verwaltung (Bogumil 2010: 78) nach geboten, größtmögliche Verwaltungskongruenz bei der Abgrenzung von Nah-, Mittel- und Oberbereichen zu gewährleisten. Ein Approximieren empirisch ermittelter Versorgungsbereichsgrenzen an Verwaltungsgrenzen (Landkreise, Verwaltungsgemeinschaften usw.) ist insofern ein überaus normativer ,Eingriff'. Eine (empirisch) gleichermaßen auf zwei Zentrale Orte ausgerichtete Gemeinde sollte (normativ) daher eindeutig einem Versorgungsbereich der beiden Zentralen Orte zugeordnet werden. Eine klare Trennung zwischen empirischem Befund (Zentrale-Orte-System) und dem normativen Konzept ist ratsam, wobei gerade die begründete Abweichung vom Befund, wie das Beispiel der Verwaltungskongruenz zeigt, den raumordnerischen Steuerungswillen im Zentrale-Orte-Konzept ausmacht (Greiving/Flex/Terfrüchte 2015: 296).

Neben solchen verwaltungspraktischen Erwägungen bei der ,Abweichung' kann eine raumordnerische Erforderlichkeit einer Funktionszuweisung im Einzelfall empirisch unterfüttert attestiert werden, wenn - was vorausgesetzt wird - Zentrale-Orte-Konzepte zum Ziel haben, ,eine flächendeckende, auch unter prognostischen Gesichtspunkten langfristig tragfähige und erreichbare Versorgung und Entwicklung im Planungsraum" (Greiving/Flex/Terfrüchte 2015: 292) zu gewährleisten. „Insofern können und sollten im Einzelfall auch hinsichtlich ihrer Ausstattung gleiche Gemeinden ungleich behandelt bzw. eingestuft werden, ohne dass damit das Gebot der Systemgerechtigkeit oder der Gleichbehandlungsgrundsatz verletzt werden würden“ (Greiving/Flex/Terfrüchte 2015: 292). Vergleichsweise starke Orte im Umland noch stärkerer Orte (in der Regel in Verdichtungsräumen) sind dann die ,Leidtragenden “ einer solchen Vorgehensweise, während eher schwache Orte umgeben von noch schwächeren Orten (in der Regel in eher ländlich-peripheren Räumen) zur Gewährleistung einer flächendeckenden Versorgung in zumutbarer Erreichbarkeit wie im Raumordnungsgesetz ( $§ 2$ Abs. 2 Ziffer 3 Satz 2) gefordert - ,Nutznießer' sind.

Eine überdurchschnittliche Versorgungs-, Entwicklungsund/oder Bereichsbildungsfunktion kann somit weder für sich genommen noch in der Addition zu einem raumordnerischen Erfordernis der zentralörtlichen Funktionszuweisung führen. Allerdings ist klarzustellen, dass dieses ,raumordnerische Erfordernis“ nicht abstrakt geprüft werden kann, sondern immer nur im konkreten (Festlegungs-)Fall angesichts der ermittelten raumstrukturellen Bedingungen und der sich daraus ergebenden Anforderungen etwa an die Gewährleistung von Erreichbarkeit. Das ,,raumordnerische Erfordernis" aber kann keinesfalls über ex ante gesetzte Schwellenwerte approximiert werden.

An Zentrenverbünde/Mehrfachorte sind dabei dieselben Anforderungen wie an monozentrale Orte zu stellen, was für die drei Funktionen Zentraler Orte Folgendes bedeutet:

- Versorgungsfunktion: Bei der Bestimmung der Versorgungsfunktionen werden die in den Teilorten lokalisierten zentralen Einrichtungen aufaddiert. Nur so kann eine Gleichbehandlung kooperierender Orte in der empirischen Überprüfung gewährleistet werden.

- Entwicklungs-/Stabilisierungsfunktion: Entwicklungsfördernde und entwicklungshemmende Faktoren werden bezogen auf das Aggregat der Teilorte bestimmt. Komplementäre Stärken (wenn etwa ein Teilort als Arbeitsmarktzentrum fungiert und der andere als Verwaltungszentrum und beide einen gemeinsamen Versorgungsbereich versorgen) sind zwar bei der Beurteilung, ob eine gemeinsame Funktionswahrnehmung im Sinne einer tatsächlichen Funktionsteilung überhaupt zweckmäßig ist, relevant. Zur Wahrung der Systemgerechtigkeit sind solche Stärken und Schwächen jedoch innerhalb des Mehrfachortes ,zu verrechnen“. Eine hohe Arbeitslosigkeit im einen Ort und eine geringe im anderen führen in der Gesamtbetrachtung zu einer mittleren Arbeitslosigkeit. In großen Einheitsgemeinden erfolgt eine solche Verrechnung bereits im Rahmen der kommunalen Statistik.

- Bereichsbildungsfunktion: Beide Teilorte müssen aus dem gemeinsamen Versorgungsbereich heraus erreichbar sein. Dies schließt die Erreichbarkeit der Teilorte untereinander zwangsläufig mit ein. Insofern verwundert es auch, dass es in einzelnen Bundesländern Zentrale Orte in Funktionsteilung gibt, die untereinander nicht in der als zumutbar erachteten Zeit erreichbar sind oder deren Festlegung gar als geeignet zur Verbesserung von Erreichbarkeiten angesehen wird. Die Umlandwirkung oder die ausgeübte zentralörtliche Gravitationskraft ist den Ausführungen zur Versorgungsfunktion folgend auf das Aggregat aus den Teilorten zu beziehen, weshalb räumliche Verflechtungen ebenfalls aufaddiert werden müssen. 


\section{Allgemeine methodische Überlegungen}

Eine empirische Fundierung von Zentrale-Orte-Konzepten muss - nach Auffassung der Verfasser - die drei vorgenannten Funktionen Zentraler Orte (Versorgung, Entwicklung/Stabilisierung und Bereichsbildung) ebenso berücksichtigen wie das raumordnerische Erfordernis. Allerdings - und das ist eines der Kernanliegen dieses Beitrags - muss die Frage geklärt werden, worum es sich bei der empirischen Fundierung wissenschaftstheoretisch überhaupt handelt; denn es ist mindestens zu unterscheiden zwischen Monitoring, Evaluation, Controlling und Validierung, wobei alle Ansätze den Bewertungs- und Entscheidungsmethoden (vgl. Scholles 2005) zuzurechnen sind. Den Landesplanungen bleiben allein deshalb schon unterschiedliche Möglichkeiten, ihre Zentrale-Orte-Konzepte auf eine solide empirische Basis zu stellen.

\subsection{Monitoring, Evaluation und Controlling}

Jacoby (2011: 547) unterscheidet zwischen Monitoring ${ }^{6}$ als Instrument zur Überwachung von Planungswirkungen, Evaluation ${ }^{7}$ als Bewertung des Planungserfolges und Controlling als Steuerung durch Planfortschreibung. Explizit weist er darauf hin, dass ,,jenseits des Monitoring und der Evaluation [...] der politische Entscheidungsprozess [liegt], in dem unter Einbeziehung der Ergebnisse des Monitoring bestimmte Handlungsoptionen entwickelt und durch Machtausübung durchgesetzt werden“" (Jacoby 2011: 548). Fürst (2010: 145) hat ein ähnliches Begriffsverständnis und stellt fest, dass Monitoring primär der Wirkungskontrolle diene, wohingegen Controlling die zielorientierte Kontrolle und Steuerung unterstütze; werden also „Veränderungen mit Zielfestlegungen verglichen, wandelt sich Monitoring in Controlling". Im Zusammenhang mit Zentrale-OrteKonzepten sind insbesondere die von Jacoby (2011: 548) beschriebenen Funktionen „Beobachtung“ und „Kontrolle“ relevant, wobei Beobachtung auf „die (laufende) Erfassung des Zustands bzw. der Entwicklung" abzielt und Kontrolle auf den „Vergleich zwischen dem Ist-Zustand bzw. der tatsächlichen Entwicklung [...] und dem Soll-Zustand bzw. der beabsichtigten Entwicklung". Die Beobachtung erfolgt dabei allein auf der Sachebene, wohingegen die Kontrolle eine Bewertung der Befunde des Soll-Ist-Vergleichs bedeutet.

Die Aufgabe der Evaluation wiederum liegt anders als beim Controlling ,nicht in der ständigen Überwachung von Prozessen [...], sondern darin, zu bestimmten Zeitpunkten das Leistungsniveau und den Zielerreichungsgrad [...] zu prüfen“ (Fürst 2010: 145). Sowohl Controlling - als Ins-

\footnotetext{
6 Vgl. zum Begriff auch Birkmann (2005).

7 Vgl. zum Begriff auch Wollmann (2005).
}

trument zur laufenden Überwachung - wie auch Evaluation - als Instrument zur punktuellen Überwachung - greifen meist auf Monitoringergebnisse zurück. Während ein Monitoring also auch ohne politisch-planerische Konsequenzen bleiben kann, wie etwa das Zentrale-Orte-Monitoring des BBSR (vgl. Einig/Zaspel-Heisters 2016), welches systematisch die Zentralen Orte in den Bundesländern erfasst, verlangt Evaluation und Controlling, so Fürst (2010: 169) „Handlungsfolgen: entweder lernt man aus den Fehlern oder man bestraft schuldhaftes Fehlverhalten“. Allerdings, und auch das konstatiert Fürst (2010: 171), ,hat die Raumplanung bei einer Reihe von Zielen häufig keinen Einfluss auf die Kräfte, welche die Zielabweichung in der Praxis bewirken, so dass eine Evaluierung für die Planung nur begrenzte Aussagekraft hat. Man kann zwar Zielverluste konstatieren, aber die Raumplanung kann sie auch künftig nicht verhindern."

Die vorgenommene Unterscheidung zwischen Monitoring, Evaluation und Controlling sei am Beispiel der Versorgungsfunktion kurz erläutert. Im Rahmen eines Monitorings können all jene Güter und Dienstleistungen landesweit erhoben werden, die landesplanerisch als zentralörtlich relevant erachtet werden. Ob nun Zentrale Orte einer bestimmten Stufe die für sie relevanten Angebote tatsächlich vorhalten (Soll-Ist-Abgleich), wäre jedoch bereits Teil einer Evaluation oder eines laufenden Controllings. Eine festgestellte Diskrepanz zwischen Soll- und Ist-Zustand müsste wiederum politisch-planerisches Eingreifen zur Folge haben, wie etwa eine Abstufung des Zentralen Ortes oder dessen finanzielle Stärkung bei fehlender (aber politisch gewollter bzw. raumordnerisch erforderlicher) Funktionserfüllung.

Vor allem Evaluation genießt mittlerweile eine hohe Bedeutung in der Planung, unter anderem „,weil man in ihr eine Legitimationsgrundlage für Maßnahmen sieht, die mit öffentlichen Geldern finanziert werden (sollen) bzw. die die Allgemeinheit betreffen“ (Döring/Bortz 2016: 976). Evaluation kann damit zur Akzeptanzsteigerung von Planungsprozessen und Planfestlegungen beitragen. Voraussetzung ist allerdings, dass klare und messbare Bewertungskriterien definiert werden, schließlich haben die gewählten Kriterien wesentlichen Einfluss auf das Ergebnis. Solche Kriterien seien unbrauchbar, so Döring und Bortz (2016: 983), „wenn sie sich nicht empirisch erfassen lassen oder wenn sie inhaltlich nicht zur Lösung des Evaluationsproblems beitragen“ (vgl. DeGEval 2008). Das raumordnerische Bestimmtheitsgebot steht somit in direktem Zusammenhang mit den wissenschaftlichen Anforderungen an Monitoring und Evaluation. Hinzu kommt - und das ist im Zusammenhang mit dem Theoriedefizit bei der Entwicklungsfunktion bereits angesprochen worden (vgl. Terfrüchte 2015: $248 \mathrm{f}$.) ,,- ,dass neben der Definition der Evaluationskriterien noch die jeweiligen Bewertungsstandards festzulegen sind. Somit 
stellt sich die Frage: Welche Ausprägungen des Evaluationskriteriums sollen als positives, ausreichendes oder negatives Ergebnis gewertet werden? Standards können z.B. als Mindest-, Regel- oder Maximalstandards gefasst werden. Sie können empirisch relativ zu einem Ausgangs- oder anderen Vergleichszustand formuliert sein [...] oder auch als absolute Werte bzw. Idealnormen festgelegt werden“ (Döring/Bortz 2016: 983). Im Zusammenhang mit der Zentralitätsforschung wird daher auch oftmals zwischen absoluter und relativer Zentralität unterschieden (Flex 2015: $235 \mathrm{f}$; Terfrüchte 2015: 119f.). Monitoring, Evaluation und Controlling sind also alles andere als trivial. Hinzu kommt das Phänomen der Pseudoevaluation, wenn die Evaluation ,für vordefinierte Zwecke instrumentalisiert bzw. missbraucht [wird]: Bei einer solchen Pseudoevaluation wird gar nicht ergebnisoffen eine wissenschaftliche Bewertung des Evaluationsgegenstandes vorgenommen, sondern das Ergebnis strategisch beeinflusst, manipuliert oder ignoriert“ (Döring/ Bortz 2016: 987). In der Praxis der Raumordnung könnte auch von ,Gefälligkeitsgutachten " gesprochen werden.

Davon zu unterscheiden sind Evaluationen, die nicht am Ende einer Planung, einer Maßnahme oder eines Planungsprozesses ansetzen, sondern ,im Sinne eines Regelkreissystems“ (Döring/Bortz 2016: 989) bereits ex ante zum Tragen kommen und das Evaluationsergebnis die Planung unmittelbar beeinflusst. Planungstheoretisch ordnet Jacoby (2011: 554) Ex-ante- und Ex-post-Evaluationen daher auch wie folgt ein: „Ex-post-Evaluationen entsprechen dem Modell der rationalen Planung mit entsprechend linearen Planungsprozessen, während planungsbegleitende Evaluationen als Teil eines systemorientierten kybernetischen Planungsmodells fungieren, bei dem Planung und Realisierung als kontinuierlicher, iterativer Handlungsprozess mit regelmäßigen Rückkopplungsschleifen begriffen wird." Das bedeutet, dass bereits im Planungsprozess mögliche Entscheidungen im Sinne von „Was wäre wenn...?“Szenarien ex ante evaluiert werden. Diese Ergebnisse können dann wiederum im weiteren Planungsprozess und der politischen Entscheidungsfindung aufgegriffen werden. Im Zusammenhang mit der Fortschreibung oder Neuaufstellung von Zentrale-Orte-Konzepten können etwa die Wirkungen unterschiedlicher Erreichbarkeitsstandards oder als relevant erachtete Einrichtungen der Daseinsvorsorge im Modell ,simuliert' werden. Gleichwohl birgt ein solches iteratives Vorgehen auch die Gefahr, dass normative planerische Festlegungen im Sinne eines gewünschten Zielzustands zu sehr am deskriptiven Befund (Ist-Zustand) ansetzen, das heißt, es werden etwa Erreichbarkeits- oder Ausstattungsstandards definiert, bei denen gegenwärtig oder absehbar keine Defizite zu erwarten sind. Die Wertebene würde dann unzulässig der Sachebene angepasst, denn: „Wollte man aus Sachaussagen normative Aussa- gen ableiten, beginge man einen Sein-Sollens-Fehlschluss (naturalistischen Fehlschluss)“" (Scholles 2001: 139).

Ein solches iteratives Vorgehen bei der Evaluation wird wiederum erleichtert, wenn kurzfristig relevante Daten etwa zu bestehenden Versorgungsstrukturen oder Verflechtungsbeziehungen bestenfalls als Zeitreihen vorliegen. Schmidt und Konze (2011: 469) schlagen daher vor, ein dauerhaftes Monitoring aller wesentlichen Elemente von Raumordnungsplänen anzustreben. Insbesondere gehe es darum, „frühzeitig zu erkennen, ob die Steuerungselemente des Plans die gewünschte Wirkung erzielen, um ggf. nachjustieren zu können" (Schmidt/Konze 2011: 469). Zudem würden sich aus einem systematischen Monitoring „Rückschlüsse für eine Weiterentwicklung des Raumordnungsplans ergeben" (Schmidt/Konze 2011: 469). Monitoring wird dann insbesondere eine „Entscheidungsunterstützungsfunktion durch Bereitstellung von entscheidungsrelevanten Informationen“ (Jacoby 2011: 559) zuteil.

Bei aller Befürwortung von Monitorings (und darauf aufbauenden Evaluationen oder Controllings) weist Fürst (2010: 169) jedoch auf zwei wesentliche Probleme hin: Erstens enthalte ein Monitoring ,Schieflagen, wenn die Indikatoren das zu beobachtende Handlungsfeld einseitig abbilden (z.B. nur in den Feldern, wo etwas zu operationalisieren ist, während nicht-operationalisierte Bereiche eher vernachlässigt werden)“ und zweitens seien ,nicht alle erforderlichen Informationen [...] zugänglich, teilweise, weil Geheimhaltungspflichten bei den Fachplanungen bestehen, teilweise, weil die Erfassung der Informationen zu aufwändig und kostenintensiv ist“".

\subsection{Validierung}

Ein vierter Zugang zur empirischen Fundierung ist, neben den - oft unmittelbar in Zusammenhang stehenden - Zugängen des Monitorings, der Evaluation und des Controllings, die Validierung. Wissenschaftstheoretisch ist Validität insbesondere als wesentliches Gütekriterium neben Objektivität und Reliabilität zu sehen. Die folgenden Ausführungen machen deutlich, dass Validierung nicht parallel oder ergänzend zu den drei vorgenannten Zugängen zu verstehen ist, sondern als Baustein einer Evaluation oder eines Controllings. Im Rahmen eines Monitorings wiederum können die für die Validierung erforderlichen Daten erhoben und bereitgestellt werden.

Ein Zentrale-Orte-Konzept ist dann valide, wenn die empirische Überprüfung zu einem - annähernd gleichen - Ergebnis kommt, das empirisch beschreibbare Zentrale-OrteSystem somit möglichst deckungsgleich mit dem normativen Konzept ist. Eine Diskrepanz zwischen Soll- und IstZustand negiert die Validität des Konzepts nicht, solange diese Diskrepanz auf normativen Erwägungen beruht, die ihren Niederschlag in einem politisch legitimierten Ziel- 


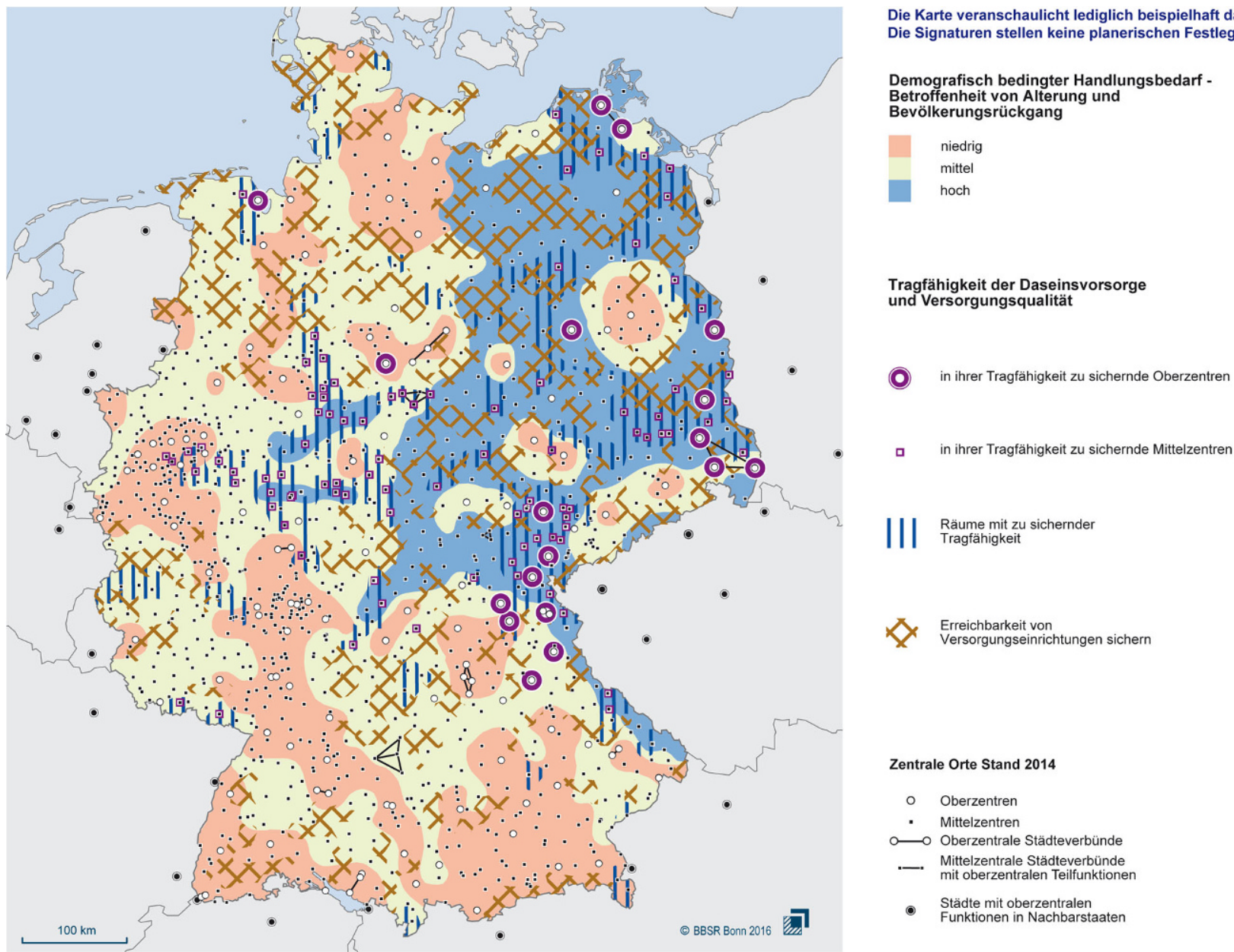

Abbildung 1 Leitbildkarte „Daseinsvorsorge“. (Quelle: MKRO 2016b: 16)

system (des Landesraumordnungsplans) finden; das verfolgte Ziel muss schließlich zum Zeitpunkt der Validierung bzw. der Evaluation nicht zwangsläufig schon erreicht sein. Wenn etwa mehrere Orte gemeinsam die Funktion eines Zentralen Ortes wahrnehmen sollen, braucht es in der Regel einige Zeit, bis eine fruchtbare Kooperation zwischen den jeweiligen Teilorten auch empirisch beobachtet werden kann. Soll ein Ort (oder ein Teilraum) über eine Funktionsfestlegung bewusst gestützt werden, sind hier andere (abgesenkte) Anforderungen an dessen Tragfähigkeit anzulegen. Sind diese Voraussetzungen hingegen nicht erfüllt, ist von nicht aus dem Zielsystem ableitbaren und damit willkürlichen Erwägungen auszugehen.

Wenn es um die Validität einer Methode bzw. eines Modells zur Beschreibung eines Zentrale-Orte-Systems mit dem Fokus auf der Zentralität von Orten geht, ,geht es also um die Güte der Operationalisierung des interessierenden Merkmals. Es ist zu klären, ob ein Test eine gelungene Operationalisierung derjenigen latenten Variable darstellt, die gemessen werden soll“" (Sedlmeier/Renkewitz 2013: 75). Das bedeutet, dass das Zielsystem im Raumord- nungsplan zunächst einer (ausreichenden) Operationalisierung zugänglich sein muss, was unter Beachtung des Bestimmtheitsgebotes eigentlich vorausgesetzt werden müsste. Bezogen auf Zentrale Orte muss somit aus dem Zielsystem heraus deutlich werden, was das latente Merkmal „Zentralität" inhaltlich ausmachen soll. Neben einer Ausstattung mit zentralörtlich relevanten Einrichtungen (Versorgungsfunktion) - ein entsprechender Katalog müsste Teil des Zielsystems sein - wären dies die Mitversorgung weiterer (Umland-)Gemeinden (Bereichsbildungsfunktion) und die Bedeutung als Entwicklungsschwerpunkt in einem Teilraum (Entwicklungs- und Stabilisierungsfunktion). Zudem sind, falls derartige - raumstrukturell bedingte - Differenzierungen beim Merkmal Zentralität vorgesehen sind, auch dafür sachlich und räumlich bestimmbare Merkmale Bestandteil des Zielsystems (etwa um zu stabilisierende periphere Räume auch bestimmen zu können).

Eine geeignete Methode zur Messung von Zentralität und damit zur Validierung eines bestehenden Zentrale-Orte-Konzepts bzw. -Systems muss daher - in Anlehnung an Sedlmeier und Renkewitz (2013: 76 ff.) - folgende Anfor- 
derungen erfüllen, aus denen sich wiederum Anforderungen an die Raumordnungspläne ableiten lassen: Inhaltsvalidität, Kriteriumsvalidität und Konstruktvalidität.

- Inhaltsvalidität: Zunächst müssten möglichst alle manifesten (beobachtbaren) Merkmale identifiziert werden, die das eigentlich interessierende, aber latente Merkmal Zentralität beschreiben können. Der Plangeber muss also sicherstellen, dass Zentralität im Zielsystem hinreichend (sachlich) bestimmt oder bestimmbar ist. Dies wird praktisch kaum möglich sein, da nicht jede normative Erwägung bei der Aufstellung von Zentrale-Orte-Konzepten auch eine empirische Grundlage hat bzw. haben kann. Dennoch sollte sich zumindest ein Großteil dessen, was sich der Plangeber unter Zentralität vorstellt, auch empirisch beobachten lassen. Es stellt sich daher auch die Frage, warum häufig allein auf (einzelne) Proxy-Indikatoren wie die Einwohnerzahl oder „Beschäftigte im Einzelhandel“ zurückgegriffen wird (Terfrüchte 2015: 93). Dass ein gewisser statistischer Zusammenhang besteht, soll dadurch nicht negiert werden. Im übertragenen Sinn geht es aber darum, ob etwa ein Intelligenztest nicht eigentlich die Konzentrationsfähigkeit misst (vgl. Sedlmeier/Renkewitz 2013: 75).

- Kriteriumsvalidität: Das Ergebnis der Zentralitätsmessung sollte eine gewisse Übereinstimmung mit dem aufweisen (sogenanntes Kriterium), was mit anderen Methoden zur Zentralitätsmessung ermittelt wurde (interne oder innere Validierung) und was etwa der Meinung von Experten oder im konkreten Fall mitunter auch der Vorstellung des Plangebers als Normgeber entspricht (externe Validierung). Eine Methode zur Zentralitätsmessung kann also zwangsläufig nur dann das Erfordernis der Kriteriumsvalidität erfüllen, wenn Zwischenergebnisse - etwa eines Gutachtens - mit dem Plangeber rückgekoppelt werden. Eine ausschließlich wissenschaftliche Lösung kann es damit de facto nicht geben, schließlich gibt es kein Naturgesetz über Zentrale Orte (anders als etwa bei der Masse von Körpern) als mögliches hinzuziehbares Vergleichskriterium.

- Konstruktvalidität: „Können mit einem Test möglichst viele Hypothesen über ein Merkmal bestätigt werden, so spricht dies für die Konstruktvalidität des Tests. [...] Je mehr Hypothesenüberprüfungen erfolgreich verlaufen sind, desto überzeugender ist die Annahme, der Test sei valide" (Sedlmeier/Renkewitz 2013: 78). Eine Methodik zur Zentralitätsmessung, die etwa nur in einem Bundesland zu validen Ergebnissen (im Sinne von Kriteriumsvalidität) führt, in einem anderen aber zu eher absurden Befunden, würde insofern das Kriterium der Konstruktvalidität nicht erfüllen.

\subsection{Beispiel: Leitbildkarte „Daseinsvorsorge sichern“}

Das von der Ministerkonferenz für Raumordnung 2016 novellierte und bekräftigte Leitbild „Daseinsvorsorge sichern“ konkretisiert die räumliche Umsetzung der Gewährleistung gleichwertiger Lebensverhältnisse durch Dienstleistungen und Infrastrukturen der Daseinsvorsorge, die regelmäßig in den Zentralen Orten gebündelt auftreten. Zur Gewährleistung müssen die Einrichtungen als Träger der Daseinsvorsorge für alle Bevölkerungsgruppen erreichbar sein. Das gilt auch für ländlich-periphere Regionen. Die entsprechende Leitbildkarte (MKRO 2016b: 16; vgl. Abbildung 1) sieht insbesondere in solchen Räumen Handlungsbedarfe hinsichtlich der Sicherung der Tragfähigkeit spezifischer Einrichtungen (und der Zentralen Orte als räumliche Bündelungspunkte dieser Einrichtungen), die sich in der näheren und mittleren Zukunft deutlichen demographischen Herausforderungen gegenübersehen.

Die Leitbildkarte kann als eine jenseits von Zielsystemen erfolgte Evaluation verstanden werden. Denn der dort definierte Soll-Zustand für den Soll-Ist-Vergleich folgt nicht den landesplanerischen Zielsystemen, sondern primär wissenschaftlichen Erwägungen. Handlungserfordernisse aus einer Diskrepanz zu einem nicht legitimierten Soll-Zustand abzuleiten, ist zumindest aus wissenschaftlich-methodischer Sicht fragwürdig (vgl. auch Flex 2015).

Es bestehen einige Probleme im Detail. So bleibt völlig unklar, auf welche zentralen Einrichtungen sich die Tragfähigkeit bezieht. Es wird pauschal unterstellt, dass wie auch immer identifizierte oder festgelegte mittelzentrale bzw. oberzentrale Einrichtungen ab einer bestimmten Einwohnerzahl in einem wie auch immer identifizierten oder festgelegten Verflechtungsbereich pauschal tragfähig seien, wobei die gewählten Einwohnerschwellenwerte nicht begründbar sind, da sie weder aus den legitimierten Zielsystemen der Länder stammen, noch das Ergebnis einer empirischen Überprüfung von Tragfähigkeiten darstellen.

Dazu sei Folgendes angemerkt: Erstens ist Raumordnung vor allem Aufgabe der Länder und grundsätzlich normativ. Das bedeutet, dass Einstufungskriterien für Zentrale Orte sowie Tragfähigkeits-, Erreichbarkeits- und Abgrenzungskriterien für Versorgungs-/Verflechtungsbereiche - bei aller ohnehin bestehenden Kritik an Einwohnerschwellenwerten - länderspezifisch und nicht bundeseinheitlich zu betrachten sind (zum Vergleich der länderspezifischen Festlegungen vgl. auch Greiving/Winkel/Flex et al. 2014: $41 \mathrm{ff}$.). Zweitens greift die Leitbildkarte die - hinsichtlich des Leitbilds „Daseinsvorsorge sichern“ - eigentlich interessierende Versorgung der Bevölkerung überhaupt nicht auf. Völlig unverständlich ist etwa, warum im südöstlichen Ruhrgebiet Mittelzentren zu sichern seien, wo doch in unmittelbarer Nachbarschaft und damit vermutlich in zumutbarer Erreichbarkeit alternative Versorgungsmöglichkeiten beste- 
hen. Gleiches gilt für (funktionsteilige) Oberzentren im Osten Sachsens oder im Nordosten Bayerns. Vielmehr müsste der Handlungsbedarf doch darin bestehen, das jeweilige Zentrale-Orte-Konzept im Hinblick auf eine - auch unter prognostischen Gesichtspunkten - Gewährleistung der Versorgung mit zentralörtlich relevanten Gütern und Dienstleistungen in zumutbarer Erreichbarkeit zu entschlacken. Das wiederum erfordert, nur dort Mittel- und Oberzentren zu ,sichern', wo sie auch tatsächlich raumordnerisch erforderlich sind. Und das sind sie nur dort, wo die Versorgung innerhalb einer zumutbaren Erreichbarkeit nicht durch „Dritte“ erfolgen kann. Und eben dieses raumordnerische Erfordernis lässt sich nicht über abstrakte Schwellenwerte approximieren.

In diesem Zusammenhang wäre zudem die alternative Option der Verbesserung der Erreichbarkeit zu diskutieren. Insofern hilft auch das angewandte Erreichbarkeitsmodell nicht weiter, da die bestehenden Zentralen Orte als Ziele und die bestehenden Mittelbereiche als Quellen verstanden werden. Vielmehr sollte die tatsächliche Versorgung der Bevölkerung im Vordergrund stehen und die erfolgt mitunter auch durch Orte, die bislang nicht als Zentrale Orte ausgewiesen sind oder auch durch andere ,zentrale Orte als die ,normativ ' gewünschten. Zudem ist hier der Einfluss der unterschiedlichen Einwohnerschwellenwerte für Mittelzentren bzw. Mittelbereiche zu sehen. So bestehen alleine deshalb keine Erreichbarkeitsprobleme in Hessen, weil dort vergleichsweise niedrige Einwohnerschwellenwerte verwendet werden und eine entsprechende Netzdichte an Mittelzentren besteht (ganz im Gegensatz etwa zu Sachsen-Anhalt). Daher ist die Leitbildkarte nur bedingt aussagekräftig für die Ableitung tatsächlicher Erreichbarkeitsdefizite.

Was für die ,in ihrer Tragfähigkeit zu sichernde[n] Ober- und Mittelzentren“ ausgeführt wurde, gilt auch für die „Räume mit zu sichernder Tragfähigkeit“ (vgl. Abbildung 1). In der Argumentation heißt es, die räumliche Dimension werde so besser deutlich als bei der punktuellen Betrachtung der Zentralen Orte selbst. Zunächst sei hierzu angemerkt, dass bereits bei der Beurteilung der Tragfähigkeit der Zentralen Orte die - theoretisch - zu versorgende Bevölkerung im Mittelbereich den Maßstab der Beurteilung gebildet hat. Eine ,deutlichere Hervorhebung ' kann schon deshalb gar nicht erfolgen. Viel problematischer ist aber die Aussage, die dahintersteckt. Denn es wird suggeriert, dass es nur ausreichend vieler Einwohner in einem Mittelbereich bedarf, damit Daseinsvorsorge ,tragfähig“ gewährleistet werden kann. Dass Tragfähigkeit sich erstens auf jede einzelne Einrichtung bezieht und diese sich zweitens zwischen den Teilräumen und Raumstrukturen mitunter deutlich unterscheiden kann, wird ohne Kommentar negiert. Das Zusammenlegen von Mittelbereichen führt also mitnichten zu einer Verbesserung der Tragfähigkeit, sondern - im Gegenteil - zu einer Verschlechterung, da nunmehr Tragfähigkeit unterstellt wird (ausreichend Einwohner im Mittelbereich), viele Einrichtungen für sich genommen aber de facto gefährdet sind, da sie - normativ gewünscht - um dieselben Einwohner konkurrieren. Auch die Festlegung von Städteverbünden geht nicht notwendigerweise mit einer verbesserten Erreichbarkeit einher, da alle Teilorte aus dem gesamten gemeinsamen Bereich erreicht werden müssen, womit der (erreichbare) Bereich automatisch deutlich kleiner ausfallen muss als bei monozentralen Orten.

Ein Konzept zur Gewährleistung von Tragfähigkeit unter Beachtung zumutbarer Erreichbarkeit muss vielmehr an einem neuen Selbstverständnis der Raumordnung als räumliche Gesamtplanung ansetzen und die - die Einrichtungen der Daseinsvorsorge vielfach steuernden - Fachplanungen koordinieren und tatsächlich an die Ziele der Raumordnung binden.

Schließlich muss an dieser Stelle auch darauf hingewiesen werden, dass die zugrunde gelegten Mittelbereiche nicht flächendeckend den - normativen - Festlegungen in den Raumordnungsplänen der Länder entsprechen, sondern in Fällen, in denen die Länder keine Mittelbereiche festgelegt haben, auf historische Festlegungen (in Nordrhein-Westfalen von 1979) oder hypothetische Festlegungen des BBSR zurückgegriffen wurde (vgl. auch BBSR 2012: 60). Tragfähigkeitsprobleme von Mittelbereichen dort zu unterstellen, wo es de facto keine Mittelbereiche gibt, ist als überaus fragwürdig anzusehen.

\section{Idealtypische Vorgehensweisen zur empirischen Fundierung}

Die in Kapitel 2 dargestellten Funktionen Zentraler Orte und in Kapitel 3 erläuterten methodischen Anforderungen an eine empirische Fundierung Zentraler Orte werden in diesem Kapitel zu idealtypischen Vorgehensweisen zusammengeführt, die sowohl wissenschaftstheoretische, raumplanungsrechtliche als auch planungspraktische Erwägungen berücksichtigen. Kern dieses Kapitels ist das Aufzeigen und Diskutieren von ,Wegen', wie die Landesplanungen grundsätzlich methodisch vorgehen können, um eine aus Gründen der Rechtssicherheit bzw. Begründbarkeit erforderliche Evidenzbasis für ihre Zentrale-Orte-Konzepte zu gewährleisten. Das Erfordernis einer solchen Diskussion folgt der gutachterlichen Tätigkeit der Verfasser in vielen Bundesländern und den damit verbundenen Erfahrungen, dass sich die Landesplanungen offenbar schwertun, sich erstens überhaupt mit einer empirischen Fundierung auseinanderzusetzen und diese dann zweitens auch auf der politischen Ebene durchzusetzen. Letzteres gilt vor allem dann, wenn im Ergebnis der Überprüfung möglicherweise 
Funktionsverluste (Abstufungen) für bestehende Zentrale Orte stehen.

Jedwede empirische Überprüfung raumordnerischer Festlegungen kann nur an den Plansätzen (Ziele und Grundsätze der Raumordnung sowie die zugehörigen Erläuterungen und Begründungen) in den Plänen und Programmen der Länder bzw. den diesen zugrunde liegenden Landesplanungsgesetzen ansetzen. Eine Fundierung losgelöst vom Planwerk ist kaum zweckmäßig (vgl. das Beispiel der Leitbildkarte in Kapitel 3). Die Ausgestaltung und vor allem der Konkretisierungsgrad der Plansätze hat eine hohe Relevanz für die Validierung (Wiechmann/Beier 2004: 388). Im Zusammenhang mit dem Bestimmtheitsgebot weist Fürst (2010: 171) zu Recht darauf hin, dass ,,nur wenige Ziele so präzise definiert [sind], dass ihre Umsetzung wirklich prüfbar wäre. Viele Ziele haben eher den Charakter von Grundsätzen, d.h. sie sind auslegungsbedürftig oder gelten nur unter bestimmten Bedingungen und hängen in ihrer Realisierung von Abwägungsprozessen in der konkreten Situation $a b$ “. Je geringer der Konkretisierungsgrad, desto eher kann ein Zentrale-Orte-Konzept - in Abhängigkeit von der gewählten Methodik - als valide und somit empirisch belastbar gelten bzw. mit Verweis auf die oben genannte Pseudoevaluation belastbar gemacht werden.

Idealtypisch lassen sich zwei Pole unterscheiden, zwischen denen sich praktisch eine Fortschreibung landesplanerischer Festlegungen vollzieht. Der eine Pol wäre ein unverändertes Festhalten am bestehenden Zielsystem, der andere bildet eine vollständige Neuaufstellung ab.

\subsection{Unverändertes Festhalten am Zielsystem}

Monitoring, Controlling und Evaluation können sich durchaus ausschließlich auf ein bestehendes landesplanerisches Zielsystem beziehen. Die Beachtung des Bestimmtheitsgebotes vorausgesetzt, besteht in der Regel dennoch ein nicht unerheblicher Auslegungsspielraum, was insbesondere aus dem oftmals geringen Konkretisierungsgrad entsprechender Festlegungen resultiert. Ziele der Raumordnung sind dann meist nicht abschließend bestimmt, aber eben bestimmbar. Wenn etwa Mittelzentren gemäß Landesentwicklungsplan/ -programm ein breites Spektrum an weiterführenden Bildungseinrichtungen aufweisen müssen, stellt sich bei der Operationalisierung die Frage, welche Bildungseinrichtungen überhaupt als weiterführend anzusehen sind, ab wann von einem breiten Spektrum die Rede sein kann, ob es um die Anzahl der (Schul-)Standorte, der Bildungsgänge, der Lehrkräfte oder der beschulten Schüler geht. Ebenso muss die Frage der Skalierung geklärt werden: Gibt es eine dichotome Skala mit den Polen ,breites Spektrum gegeben “ und ,breites Spektrum nicht gegeben ' oder ist eine ordinale oder metrische Skala sachgerecht, die das Ausmaß der Ausstattung mit entsprechenden Einrichtungen abbildet?
Das Ergebnis etwa der Evaluation eines bestehenden Zentrale-Orte-Konzepts wäre dann ein Soll-Ist-Abgleich, der die Einstufung der Orte im Zentrale-Orte-Konzept mit ihrer tatsächlichen Funktion im Zentrale-Orte-System abgleicht. Erfüllt ein Ort seine ihm zugewiesene Funktion nicht und ist dieser darüber hinaus raumordnerisch nicht erforderlich (etwa in Folge von Erreichbarkeitsdefiziten), müsste ein solcher Ort bei unverändertem Zielsystem seine Funktion aberkannt bekommen. Je nach Operationalisierung des Zielsystems und je nach Konkretisierungsgrad können die empirischen Befunde jedoch höchst unterschiedlich ausfallen, sodass besagtem Ort bereits bei leicht veränderten Modellannahmen weiterhin die ihm zugewiesene Funktion im Zentrale-Orte-System/-Konzept attestiert werden kann. Ist jedoch im Zielsystem konkret bestimmt, dass etwa Oberzentren eine Universität vorhalten müssen und weist ein als Oberzentrum eingestufter Ort keine Universität, sondern ,nur' eine Fachhochschule auf, würde eine empirische Überprüfung den Ort unabhängig von der weiteren Operationalisierung nicht als Oberzentrum ,bestätigen ${ }^{*}$ können. Es verwundert daher kaum, dass zahlreiche Landesplanungen im Rahmen von Fortschreibungen daher entweder (leichte) Veränderungen am Zielsystem vornehmen und etwa an aktuelle Rahmenbedingungen anpassen oder die jeweiligen Festlegungen mit Grundsatz- statt mit Zielcharakter vornehmen, womit ein gewisser Abwägungsspielraum verbleibt.

\subsection{Vollständige Neuaufstellung des Zielsystems}

Die vollständige Neuaufstellung des landesplanerischen Zielsystems bedeutet zwar nicht zwangsläufig eine ,tabula rasa'. Dennoch gibt es praktisch keinen ,Bestandsschutz', das heißt, Funktionszuweisungen, die aus früheren Zielen oder Grundsätzen der Raumordnung resultieren, genießen kein besonderes Gewicht bei der Erarbeitung eines neuen Zentrale-Orte-Konzepts. Sowohl Ideen aus Planung, Politik und Gesellschaft wie auch aus der Wissenschaft können in den Prozess der Neuaufstellung einfließen. Während etwa die ersten Zentrale-Orte-Konzepte stets von der grund- bis zur oberzentralen Hierarchiestufe reichten, haben infolge der politisch-planerischen und wissenschaftlichen Auseinandersetzung mit Metropolregionen einige Landesplanungen das Zentrale-Orte-Konzept um eine metropolitane Hierarchiestufe oberhalb der Oberzentren ergänzt (z. B. Berlin-Brandenburg) oder planen eine Ergänzung (z. B. Bayern). Eine vollständige Neuaufstellung von ZentraleOrte-Konzepten kann zwar aus raumordnungspolitischen Erwägungen heraus zweckmäßig sein, stößt aber in der Regel auf großen kommunalen Widerstand, insbesondere wenn eine Abstufung als Folge eines Funktionsverlustes im Rahmen der Neuaufstellung droht. Die bayerische Landesplanung hat daher auch mit der Teilfortschreibung des 
Landesentwicklungsprogramms 2016 einen landesweiten Bestandsschutz im Sinne einer Nicht-Abstufungsgarantie ausgesprochen.

\subsection{Iterative Fortschreibung des Zielsystems}

Zwischen diesen Polen - und das wäre die dritte mögliche Vorgehensweise - ist jedoch auch ein iteratives Vorgehen möglich. Eine iterative Fortschreibung oder Neuaufstellung des landesplanerischen Zielsystems bewegt sich zwischen den vorgenannten Polen und erscheint den Verfassern aufgrund ihrer planungspraktischen Erfahrung der zielführendste, weil umsetzbare und zugleich wissenschaftlichen Anforderungen genügende Weg zu sein. Ausgangspunkt einer empirischen Überprüfung ist stets das bestehende Zielsystem. Infolge der Operationalisierung und der anschlieBenden empirischen Befunde wird das Zielsystem dann in der Art angepasst, wie es den politisch-planerischen Vorstellungen entspricht. Mitunter ist es erst der empirische Befund, der für bestimmte Veränderungserfordernisse sensibilisiert. Die Einführung von Zentrenverbünden, Mehrfachorten oder Zwischenstufen im Zentrale-Orte-Konzept (wie etwa Mittelzentren mit Teilfunktionen eines Oberzentrums) können als Reaktion darauf verstanden werden, dass das Christallersche Ideal monozentraler Orte mit eindeutigen Versorgungsbereichen nur noch bedingt der empirisch beschreibbaren Realität entspricht. Im Rahmen einer iterativen Fortschreibung spielen - wenn auch den handelnden Akteuren nicht immer bewusst - daher auch Szenarien eine große Rolle. Wenn etwa Erreichbarkeitsstandards mit Zielqualität festgelegt werden sollen, kann mittels Szenarien überprüft werden, welche Standards überhaupt zweckmäBig sein können. Da die Landesplanungsbehörden vielfach nicht in der Lage sind, umfängliche empirische Arbeiten selbst zu leisten, kommt Gutachtern in solchen iterativen Fortschreibungsprozessen eine besondere Rolle zu. Während etwa bei einer ,klassischen' Evaluierung des bestehenden Zentrale-Orte-Konzepts ein Gutachten mit einem SollIst-Vergleich und gegebenenfalls abgeleiteten Handlungsempfehlungen für Politik und Planung abschließt, sind die Ergebnisse bei einem iterativen Vorgehen stets als impulsgebende Zwischenergebnisse zu verstehen. Bevor etwa landesweit die zentralörtliche Funktion von Orten empirisch überprüft wird, wäre die Operationalisierung dessen, was überhaupt zentralörtlich relevante Funktionen sind, ein erster mit der Landesplanung rückzukoppelnder Schritt. Es erfolgt dann bereits die erste Anpassung oder Konkretisierung der bestehenden Ziele.

\subsection{Empirische Fundierung jenseits eines Zielsystems}

Ein vierter - wenngleich nicht empfohlener - Zugang wiederum steht neben den oben genannten Polen bzw. kann im besten Fall eine vollständige Neuaufstellung vorbereiten: eine empirische Fundierung jenseits des Zielsystems. Zu bedenken ist dann jedoch, dass normative Momente raumordnerischer Festlegungen damit de facto negiert werden würden. Und insbesondere Fragen zumutbarer Erreichbarkeit oder der Priorisierung von Erreichbarkeit im Verhältnis zur Tragfähigkeit erfordern eine politisch-planerische Willensbildung. Rein akademisch können solche Fragen nicht geklärt werden. Dennoch seien an dieser Stelle einige Gedanken zu diesem Zugang aufgeführt.

Die ursprüngliche Arbeit von Christaller zu Zentralen Orten war - vereinfacht ausgedrückt - nicht mehr, aber eben auch nicht weniger als die Beschreibung von Regelmäßigkeiten im süddeutschen Städtesystem (vgl. Christaller 1968). Zwar war ihm der normative Gehalt seiner Arbeit durchaus bewusst (Blotevogel 2002b: $12 \mathrm{f}$.), die unmittelbare Implementierung seiner Überlegungen in die deutsche Raumordnung dürfte jedoch kaum bezweckt gewesen sein. Insofern ist es auch weiterhin denkbar, dass es vor allem wissenschaftliche Arbeiten gibt, die sich mit der Städtesystemforschung im Allgemeinen und der Zentralitätsforschung im Speziellen beschäftigen, ohne an bestehenden raumordnerischen Festlegungen anzusetzen oder konkrete Handlungsempfehlungen auszusprechen (vgl. Terfrüchte 2015). Und immer dann, wenn für die Operationalisierung normative Entscheidungen erforderlich sind, wie dies etwa bei Erreichbarkeitsstandards unumgänglich ist, werden diese hypothetisch gesetzt, als Prämissen angenommen oder rekrutieren sich etwa aus anderen Forschungsarbeiten oder Richtlinien. Für Erreichbarkeitsstandards im öffentlichen Verkehr und im Individualverkehr werden etwa oftmals die sogenannten Richtlinien für Integrierte Netzgestaltung (RIN) herangezogen. Meist greifen jedoch auch primär wissenschaftliche Arbeiten zu Zentralen Orten ein bestimmtes landesplanerisches Zielsystem auf, was nicht selten in mehr oder weniger konkreten Empfehlungen für die jeweilige Landes- oder Regionalplanung mündet (vgl. für die Planungsregion Düsseldorf Flex 2015; vgl. für RheinlandPfalz Furkert 2015). Problematisch ist vor diesem Hintergrund, wenn Monitoring und Evaluation im oben dargestellten Verständnis eben nicht an landesplanerischen Zielsystemen anknüpfen, aber dennoch in einem - für Evaluationen auch gängigen - politisch-planerischen Handlungserfordernis münden.

\subsection{Schlussfolgerungen für die Operationalisierung/ Entwicklung des raumordnerischen Zielsystems}

Die angewandte Methodik zur Evaluation oder zur Validierung von Zentrale-Orte-Konzepten muss mit dem jeweiligen Zielsystem umgehen können. Dabei ist es zunächst unerheblich, ob das Zielsystem unverändert Bestand hat oder im Fortschreibungs- bzw. Neuaufstellungsprozess ver- 
ändert wird. Wesentlich ist etwa der Umgang mit unterschiedlich vielen Hierarchiestufen (z. B. Einbeziehen der metropolitanen Ebene oder einer intrakommunalen Perspektive), die Operationalisierung über Funktionsbereiche (z. B. Bildung), Teilfunktionsbereiche (z. B. allgemeinbildende Schulen), Funktionen (z. B. Schulen der Sekundarstufe II) bis hin zu den tatsächlich beobachtbaren Merkmalen (z. B. Gymnasien, Integrierte Gesamtschulen). Und sofern Erreichbarkeits- und Tragfähigkeitsstandards nicht $e x$ ante bestimmt sind (was für Zentrale-Orte-Konzepte auch nicht zwingend erforderlich ist), diese aber Einzug in ein Zielsystem nehmen sollen, muss die Methodik auch das empirische Ableiten entsprechender Schwellenwerte ermöglichen. Dabei ist grundsätzlich zu bedenken, dass es die eine richtige Methodik nicht gibt. So haben auch Obergerichte vielfach geurteilt, dass grundsätzlich keine Methode pauschal am geeignetsten ist, sondern der Plangeber sich eine Methode zu eigen machen muss, mit der er gedenkt, zu einem sachgerechten Befund zu kommen. Vielmehr gilt es also, allgemeine methodische Gütekriterien wie Objektivität, Validität und Reliabilität zu beachten. Insofern kann Folgendes festgehalten werden:

- Die einzig richtige Methode zur Validierung von Zentrale-Orte-Konzepten gibt es nicht.

- Die einzelnen methodischen Ansätze dienen stets der Komplexitätsreduktion auf der Sachebene, sie müssen zugleich das politische Entscheidungsproblem auf diejenigen Fragen fokussieren, die der Wertebene zuzurechnen sind.

- Umso wichtiger ist daher ein frühzeitig zu initiierender Diskursprozess zwischen der Landesplanung als verfahrensführender Behörde, den Planadressaten, also vor allem der Regionalplanung, und einem gegebenenfalls beteiligten externen Gutachter, um eine Verständigung über die Wertebene und das methodische Vorgehen zur Erfassung der Sachebene zu erzielen. Auf diese Weise lassen sich Ergebnisse erzeugen, die inhaltlich überzeugen und politisch auch kommunizier- und durchsetzbar sind.

\section{Fazit}

Wie gezeigt wurde, ist die empirische Fundierung von Zentrale-Orte-Konzepten eine überaus komplexe Angelegenheit. Wenn aus obergerichtlichen Urteilen folgt, dass sich Plangeber eine - selbstgewählte - Methode zu eigen machen sollen, setzt dies voraus, dass sich die Plangeber über die methodischen Implikationen bewusst sind, schließlich müssen sie begründen, weshalb sie die eine oder andere Methode angewendet haben. Nimmt man nun den Legitimationsdruck, unter dem Politik und Planung seit einigen Jahren stehen, hinzu, kommt wissenschaftlichen Gutachten eine entscheidende Rolle zu. Döring und Bortz (2016:
988) gehen jedoch auch hinsichtlich der Evaluationsergebnisse nicht davon aus, dass diese ,auch wirklich rezipiert, verstanden und akzeptiert werden und dann auch noch zur Ableitung und Umsetzung der richtigen Konsequenzen führen“. Insofern müssten auch ,weitere Aktivitäten wie Beratung, Coaching, Workshops [...] [erfolgen], in denen Bedeutung und praktische Konsequenzen der Evaluationsergebnisse diskutiert und konzipiert werden“ (Döring/Bortz 2016: 988).

Umfangreiche Monitorings, als Selbstzweck oder als Grundlage für Evaluation oder Validierung, ermöglichen dabei grundsätzlich eine weniger vom Gutachter abhängige Datenbasis für eine empirische Fundierung. Über vielfach bestehendes Siedlungsflächenmonitoring hinaus bietet es sich somit auch an, ein umfassendes Regionalmonitoring aufzubauen, das etwa die Versorgungs- und Entwicklungs-/ Stabilisierungsfunktion von Städten und Gemeinden ebenso abbildet wie bestehende räumlich-funktionale Verflechtungen (vgl. Baumgart/David/Diehl et al. 2013: 10; Terfrüchte 2015: 248) oder die tatsächliche Umsetzung einer politisch gewollten Funktionsteilung innerhalb von Städteverbünden (vgl. auch BMVBS/BBR 2008). Der erste Schritt der vorgeschlagenen Operationalisierung liegt damit beim Plangeber selbst. Dadurch wird auch das skizzierte iterative Vorgehen bei der Fortschreibung bzw. Neuaufstellung von Zentrale-Orte-Konzepten erleichtert, da erste empirische Befunde bereits vor der Vergabe und dem Vorliegen von Gutachten vorhanden sind. Die starre Trennung zwischen dem (deskriptiven) Zentrale-Orte-System und dem (normativen) Zentrale-Orte-Konzept verwässert dadurch zwangsläufig, da der empirische Befund bereits auf normativen Erwägungen (etwa zur zumutbaren Erreichbarkeit) fußt. Je mehr Schritte in einem iterativen Vorgehen bereits erfolgt sind, desto weniger politisch-planerische Spielräume bestehen dann noch, desto näher kommt der empirische (Zwischen-)Befund dem abschließenden normativen Konzept und desto weniger methodische Ansätze sind dann noch zur Validierung des Zentrale-Orte-Konzepts geeignet. Das bedeutet dann einerseits eine zunehmende Validität der empirischen Befunde, erfordert andererseits aber auch ein frühzeitiges ,Einsteigen“ der Landesplanungen in einen solchen iterativen Prozess. Denn wenn vorab methodische Bausteine ,zementiert' sind, die nicht mit dem raumordnerischen Zielsystem korrespondieren (können), tendieren die zentralörtlichen Festlegungen zur Willkür.

In den hier vorgenommenen methodischen Schlüssen schwingen zweifelsohne und ganz in der Tradition Christallers auch normative Schlüsse mit. Gleichwohl sind diese primär darauf gerichtet, welches Vorgehen aus welchem Grund zweckmäßig erscheint. Ein Plädoyer, welche Einrichtungen etwa zur Versorgungsfunktion zählen sollten, welche Erreichbarkeitsstandards als zumutbar gelten sollten oder wie viele Hierarchiestufen ein Zentrale-Orte-Kon- 
zept aufweisen sollte, sind diese Schlüsse jedoch nicht. Dies obliegt dem politisch legitimierten Plangeber. Die bisherigen Erfahrungen in der gutachterlichen Praxis haben jedoch gezeigt, dass zumindest eine Straffung des Zentrale-OrteKonzepts vor dem Hintergrund einer flächendeckenden Daseinsvorsorge und gleichwertiger Lebensverhältnisse in vielen Fällen aus den empirischen Befunden abgeleitet werden kann, wobei die größten Anpassungserfordernisse nicht in den ländlichen, sondern in den verdichteten Landesteilen gesehen wurden. In denen sind regelmäßig Zentrale Orte zu finden, die zwar die geltenden Einwohnerschwellenwerte und häufig auch die Anforderungen aus Ausstattungskatalogen erfüllen, aber selten bereichsbildend wirken und keinesfalls zur Gewährleistung einer erreichbaren Versorgung erforderlich sind. $\mathrm{Ob}$ und wie diese Befunde normativ in ein Zentrale-Orte-Konzept überführt werden, ist Aufgabe von Politik und Planung. Die mehrfach erwähnten Zentrenverbünde bzw. Mehrfachorte sind vor diesem Hintergrund auch nicht als Empfehlung zu verstehen, auf Erreichbarkeits- oder Tragfähigkeitsdefizite mit kooperativen Lösungen (Funktionsteilung) zu reagieren. Dass sogenannte monozentrale Orte allein schon hinsichtlich entstehender Transaktionskosten die von den Verfassern bevorzugte Variante sind, wie es an anderer Stelle vorgeschlagen wurde (Winkel/Greiving 2008; Greiving/Flex/Terfrüchte 2015), soll dennoch nicht verschwiegen werden. Welche konkreten methodischen Ansätze mit welchen - aus den landesplanerischen Festlegungen resultierenden - Prämissen jeweils am besten umgehen können, kann im Rahmen eines solchen Beitrags nicht geklärt werden. Dafür sind die Ausgangskonstellationen zu unterschiedlich.

\section{Literatur}

ARL - Akademie für Raumforschung und Landesplanung (2013): Anforderungen an ein zukünftiges Zentrale-Orte-Konzept. Beispiele aus Hessen, Rheinland-Pfalz und dem Saarland. Hannover. = Positionspapier aus der ARL 92.

ARL - Akademie für Raumforschung und Landesplanung (2015): Neuaufstellung des Zentrale-Orte-Konzepts in Nordrhein-Westfalen. Hannover. $=$ Positionspapier aus der ARL 102.

Baumgart, S.; David, C.-H.; Diehl, J.; Finke, L.; van Gemmeren, C.; Greiving, S.; Kötter, K.; Konze, H.; Petzinger, T.; Terfrüchte, T.; Wiechmann, T. (2013): Planen in der Zukunft. In: Baumgart, S.; Terfrüchte, T. (Hrsg.): Zukunft der Regionalplanung in Nordrhein-Westfalen. Hannover, 5-17. = Arbeitsberichte der ARL 6.

BBSR - Bundesinstitut für Bau-, Stadt- und Raumforschung (2012): Raumabgrenzungen und Raumtypen des BBSR. Bonn. = Analysen Bau.Stadt.Raum 6.

Birkmann, J. (2005): Monitoring. In: ARL - Akademie für Raumforschung und Landesplanung (Hrsg.): Handwörterbuch der Raumordnung. Hannover, 668-674.

Blotevogel, H. H. (Hrsg.) (2002a): Fortentwicklung des Zentrale-Orte-Konzepts. Hannover. $=$ Forschungs- und Sitzungsberichte der ARL 217.

Blotevogel, H. H. (2002b): Zum Verhältnis der regionalökonomischen Zentrale-Orte-Theorie zum Zentrale-Orte-Konzept der Raumord- nung. In: Blotevogel, H. H. (Hrsg.): Fortentwicklung des Zentrale-Orte-Konzepts. Hannover, 10-16. = Forschungs- und Sitzungsberichte der ARL 217.

Blotevogel, H. H. (2005): Zentrale Orte. In ARL - Akademie für Raumforschung und Landesplanung (Hrsg.): Handwörterbuch der Raumordnung. Hannover, 1307-1315.

BMVBS - Bundesministerium für Verkehr, Bau und Stadtentwicklung; BBR - Bundesamt für Bauwesen und Raumordnung (Hrsg.) (2008): Kooperation zentraler Orte in schrumpfenden Regionen. Praxiserfahrungsstudie. Bonn. $=$ Werkstatt: Praxis 53.

Boettcher, F.; Junkernheinrich, M. (2010): Kommunaler Finanz- und Schuldenreport Nordrhein-Westfalen. Gütersloh.

Bogumil, J. (2010): Die Ebenen der Verwaltung, die Verteilung der Aufgaben und die Realität der Verwaltungspolitik. In: Schimanke, D. (Hrsg.): Verwaltung und Raum. Zur Diskussion um Leistungsfähigkeit und Integrationsfunktion von Verwaltungseinheiten. Baden-Baden, 77-88. = Schriften der Deutschen Sektion des Internationalen Instituts für Verwaltungswissenschaften 34.

Christaller, W. (1968): Die zentralen Orte in Süddeutschland. Eine ökonomisch-geographische Untersuchung über die Gesetzmäßigkeit der Verbreitung und Entwicklung der Siedlungen mit städtischen Funktionen. Darmstadt.

Czihal, T.; von Stillfried, D.; Schallock, M. (2012): Regionale Mitversorgungsbeziehungen in der ambulanten Versorgung. Berlin. http://www.versorgungsatlas.de/fileadmin/ziva_docs/21/ Mitversorgung_Bericht_upload20130304.pdf (02.06.2017).

DeGEval - Gesellschaft für Evaluation (2008): Standards für Evaluation. Mainz.

Deiters, J. (1978): Zur empirischen Überprüfbarkeit der Theorie zentraler Orte. Fallstudie Westerwald. Bonn. = Arbeiten zur rheinischen Landeskunde 44.

Döring, N.; Bortz, J. (2016): Forschungsmethoden und Evaluation in den Sozial- und Humanwissenschaften. Berlin/Heidelberg.

Einig, K.; Zaspel-Heisters, B. (2016): Das System Zentraler Orte in Deutschland. In: Greiving, S.; Flex, F. (Hrsg.): Neuaufstellung des Zentrale-Orte-Konzepts in Nordrhein-Westfalen. Hannover, 3-19. = Arbeitsberichte der ARL 17.

Faßbender, K. (2012): Rechtsgutachten zu den Anforderungen an regionalplanerische Festlegungen zur Hochwasservorsorge, erstattet im Auftrag des Regionalen Planungsverbands Oberes Elbtal/ Osterzgebirge. Leipzig.

Flex, F. (2015): Zentralörtliche Funktionsräume. Empirische Ermittlung und konzeptionelle Ausgestaltung am Beispiel der Planungsregion Düsseldorf. Dissertation, Technische Universität Dortmund.

Fürst, D. (2010): Raumplanung. Herausforderungen des deutschen Institutionensystems. Detmold. = Planungswissenschaftliche Studien zu Raumordnung und Regionalentwicklung 1.

Furkert, M. (2015): Erkennen und Handeln. Restrukturierung der landesplanerischen Mittelbereiche in Rheinland-Pfalz. Hamburg. = Geographica. Schriftenreihe Geowissenschaften und Geographie 11.

Ganser, K. (1977): Zentrale Orte und Entwicklungszentren. In: Ganser, K.; Heinritz, G.; Klingbeil, D.; Mittermaier, K.; Niedzwetzki, K.; Popp, H.; Schrettenbrunner, H. (Hrsg.): Beiträge zur Zentralitätsforschung. Kallmünz/Regensburg, 105-124. = Münchener Geographische Hefte 39.

Greiving, S.; Flex, F. (Hrsg.) (2016): Neuaufstellung des Zentrale-Orte-Konzepts in Nordrhein-Westfalen. Hannover. = Arbeitsberichte der ARL 17.

Greiving, S.; Flex, F.; Terfrüchte, T. (2015): Vergleichende Untersuchung der Zentrale-Orte-Konzepte in den Ländern und Empfehlungen zu ihrer Weiterentwicklung. In: Raumforschung und Raumordnung 73, 4, 285-297.

Greiving, S.; Winkel, R.; Flex, F.; Terfrüchte, T. (2014): Reform der Zentrale-Orte-Konzepte in den Ländern und Folgen für Siedlungsstruktur und Daseinsvorsorge. Endbericht im Auftrag des Bundesinstituts für Bau-, Stadt- und Raumforschung. Bonn. 
Heinritz, G. (1977): Einzugsgebiete und zentralörtliche Bereiche. Methodische Probleme der empirischen Zentralitätsforschung. In: Ganser, K.; Heinritz, G.; Klingbeil, D.; Mittermaier, K.; Niedzwetzki, K.; Popp, H.; Schrettenbrunner, H. (Hrsg.): Beiträge zur Zentralitätsforschung. Kallmünz/Regensburg, 9-43. = Münchener Geographische Hefte 39.

Henckel, D.; Kolleck, B.; Mittag, K.; Seidel-Schulze, A. (2002): Städtetypen in Nordrhein-Westfalen. Gutachten im Auftrag der Enquetekommission „Die Zukunft der Städte in Nordrhein-Westfalen“. Endbericht. Berlin.

Hesse, J. J. (2010): Kommunalstrukturen in Niedersachsen. Untersuchung im Auftrag des Ministeriums für Inneres und Sport des Landes Niedersachsen. Berlin.

Jacoby, C. (2011): Monitoring, Evaluation und Controlling. In: ARL - Akademie für Raumforschung und Landesplanung (Hrsg.): Grundriss der Raumordnung und Raumentwicklung. Hannover, 547-559.

Junkernheinrich, M.; Boettcher, F.; Brand, S.; Steinmüller, L.; Holler, B. (2011): Neuabgrenzung leistungsfähiger Gemeindegebiete. Ein verwaltungsökonomischer Beitrag zur Kommunal- und Verwaltungsreform von Verbandsgemeinden und verbandsfreien $\mathrm{Ge}$ meinden in Rheinland-Pfalz. Gutachten im Auftrag des Ministeriums des Innern und für Sport. Endbericht für die Untersuchungsregion 1. Kaiserslautern.

Klöpper, R. (1970): Zentrale Orte und ihre Bereiche. In: ARL - Akademie für Raumforschung und Landesplanung (Hrsg.): Handwörterbuch der Raumforschung und Raumordnung. Band III. Hannover, 3849-3860.

Klöpper, R. (1972): Die deutsche geographische Stadt-Umland-Forschung. Entwicklung und Erfahrungen. In: Schöller, P. (Hrsg.): Zentralitätsforschung. Darmstadt, 252-266. = Wege der Forschung 301.

Körber, J. (1972): Einzugsbereiche zentraler Orte. Bericht über eine Beispieluntersuchung in Rheinland-Pfalz. In: Schöller, P. (Hrsg.): Zentralitätsforschung. Darmstadt, 231-251. = Wege der Forschung 301.

LAG ARL - Landesarbeitsgemeinschaft Bremen/Hamburg/Niedersachsen/ Schleswig-Holstein der Akademie für Raumforschung und Landesplanung (2014): Stärkung der Innenstädte und Ortskerne. Initiativen und Instrumente aus der Praxis für die Praxis. Arbeitshilfe für Kommunen. Hannover.

Landtag Nordrhein-Westfalen (2015): Bewertung der Tragfähigkeit der öffentlichen Haushalte in Nordrhein-Westfalen. Unter den Bedingungen der Schuldenbremse und des demografischen Wandels in der Dekade 2020 bis 2030. Abschlussbericht der Enquetekommission III des Landtags Nordrhein-Westfalen. Landtagsdrucksache 16/9500. Düsseldorf.

Langguth, N. (2011): Die Grenzen der Raumordnungsplanung. Zur Abgrenzung der Gesetzgebungskompetenzen für Raumordnung und Bauleitplanung. In: Zeitschrift für deutsches und internationales Bau- und Vergaberecht 34, 5, 436-441.

MKRO - Ministerkonferenz für Raumordnung (2016a): Entschließung „Zentrale Orte“. Beschluss der 41. Ministerkonferenz für Raumordnung am 9. März 2016 in Berlin. Berlin.

MKRO - Ministerkonferenz für Raumordnung (2016b): Leitbilder und Handlungsstrategien für die Raumentwicklung in Deutschland. Beschluss der 41. Ministerkonferenz für Raumordnung am 9. März 2016 in Berlin. Berlin.

Niedzwetzki, K. (1977): Methoden der Zentralitätsmessung. In Ganser, K.; Heinritz, G.; Klingbeil, D.; Mittermaier, K.; Niedzwetzki, K.; Popp, H.; Schrettenbrunner, H. (Hrsg.): Beiträge zur Zentralitätsforschung. Kallmünz/Regensburg, 75-103. = Münchener Geographische Hefte, 39.

Schmidt, P. I.; Konze, H. (2011): Planaufstellungs- und -änderungsverfahren. In: ARL - Akademie für Raumforschung und Landesplanung (Hrsg.): Grundriss der Raumordnung und Raumentwicklung. Hannover, 451-472.

Scholles, F. (2001): Zielsysteme in der Planung. In: Fürst, D.; Scholles, F. (Hrsg.): Handbuch Theorien und Methoden der Raum- und Umweltplanung. Dortmund, 139-143.

Scholles, F. (2005): Bewertungs- und Entscheidungsmethoden. In: ARL - Akademie für Raumforschung und Landesplanung (Hrsg.): Handwörterbuch der Raumordnung. Hannover, 97-106.

Sedlmeier, P.; Renkewitz, F. (2013): Forschungsmethoden und Statistik für Psychologen und Sozialwissenschaftler. München.

Staack, J. (1995): Die Klassifikation deutscher Städte nach ihrer regionalen Zentralität. Frankfurt am Main. = Europäische Hochschulschriften 1751.

Terfrüchte, T. (2015): Regionale Handlungsräume. Gliederung und Einflussfaktoren am Beispiel Nordrhein-Westfalens. Lemgo. = Metropolis und Region 14.

Wiechmann, T.; Beier, M. (2004): Evaluationen in der Regionalentwicklung. Eine vernachlässigte Herausforderung für die Raumplanung. In: Raumforschung und Raumordnung 62, 6, 387-396.

Wiechmann, T.; Terfrüchte, T. (2013): Akzeptanz regionaler Planungsprozesse und -ergebnisse. In: Baumgart, S.; Terfrüchte, T. (Hrsg.): Zukunft der Regionalplanung in Nordrhein-Westfalen. Hannover, 18-39. = Arbeitsberichte der ARL 6.

Wiechmann, T.; Terfrüchte, T. (2017): Smart Country regional gedacht. Teilräumliche Analysen für digitale Strategien in Deutschland. Gütersloh.

Winkel, R.; Greiving, S. (2008): Zentrale-Orte-Konzepte in praxisorientierter Weiterentwicklung. Monozentrale Orte, Städteverbünde und zentralörtliche Funktionsräume. In: RaumPlanung 141, 263 268.

Wollmann, H. (2005): Evaluation. In: ARL - Akademie für Raumforschung und Landesplanung (Hrsg.): Handwörterbuch der Raumordnung. Hannover, 274-280. 